\title{
Theoretical study of the electronic excited states in ultrathin ionic layers supported on metal surfaces: $\mathrm{NaCl} / \mathrm{Cu}(\mathbf{1 1 1})$
}

\author{
Sergio Díaz-Tendero, ${ }^{1, *}$ Andrey G. Borisov, ${ }^{2,3, \dagger}$ and Jean-Pierre Gauyacq ${ }^{2,3, \ddagger}$ \\ ${ }^{1}$ CNRS, Laboratoire des Collisions Atomiques et Moléculaires, LCAM, Unité de Recherches CNRS-Université Paris-Sud UMR 8625, \\ Bâtiment 351, Université Paris-Sud, F-91405 Orsay Cedex, France \\ ${ }^{2}$ CNRS, Institut des Sciences Moléculaires d'Orsay, ISMO, Unité de Recherches CNRS-Université Paris-Sud UMR 8214, Bâtiment 351, \\ Université Paris-Sud, F-91405 Orsay Cedex, France \\ ${ }^{3}$ Université Paris-Sud, Institut des Sciences Moléculaires d'Orsay, ISMO, Unité de Recherches CNRS-Université Paris-Sud UMR 8214, \\ Bâtiment 351, Université Paris-Sud, F-91405 Orsay Cedex, France
}

(Received 13 October 2010; published 29 March 2011)

\begin{abstract}
We present a theoretical study of the electronic excited states in ultrathin ionic layers supported on metal surfaces. We have studied 1, 2, 3, and 4 monolayers of $\mathrm{NaCl}$ on a $\mathrm{Cu}(111)$ surface. Energies, lifetimes, and associated wave functions of the excited states have been obtained with a joint, model potential-wave packet propagation approach. The excited state with the lowest energy has the character of an image potential state repelled from the surface by the $\mathrm{NaCl}$ layer. The next two states present a mixed character of image potential states and $\mathrm{NaCl}$ layer states corresponding to the quantization of the conduction band in the finite-size layer. We discuss the role of the layer thickness in decoupling these states from the metal surface and how it affects their lifetime.
\end{abstract}

DOI: 10.1103/PhysRevB.83.115453

PACS number(s): 71.15.Qe, 73.20.Hb, 73.61.Ng

\section{INTRODUCTION}

Excited electronic states at surfaces are very often invoked in reaction mechanisms: The transient formation of an excited state, a resonance, by (photo)excitation or electronic capture can induce an internal evolution in the system that eventually leads to the reaction channel (see e.g., Ref. 1). In this context, detailed knowledge of the excited states and their properties, in particular the various parameters influencing their lifetime, is quite important. Indeed, a too short lifetime severely hampers the efficiency of a resonance-mediated reaction mechanism. For this purpose, time-resolved two-photon photoemission (TR-2PPE) is a very efficient technique for determining the time evolution of a transient excited state and its lifetime. This technique yielded a wealth of results in the field of ultrafast electron dynamics at surfaces ${ }^{2}$ and the induced phenomena. ${ }^{3-5}$ TR-2PPE experiments showed that the introduction of an ultrathin insulating layer on a surface can dramatically modify the electron dynamics by partly decoupling the excited state from the substrate. Rare-gas layers adsorbed on a metal have thus been shown to lead to a strong increase of the lifetimes of the excited states at surfaces, both in the case of states delocalized on the surface such as image potential states (ISs) $)^{6-9}$ and of states localized on adsorbates. ${ }^{10-13}$ However, the rare-gas layer does not have the same insulating role in all systems. In the case of states delocalized on the surface, it leads to (i) ISs repelled into vacuum by the insulating layer, ${ }^{9}$ (ii) the mixture of image potential states with conduction band states when the ISs are not degenerated with the insulator forbidden band, ${ }^{7,8}$ and (iii) layer states corresponding to ISs located inside a dielectric in the case of very thick layers. ${ }^{14,15}$ So, depending on the relative energy of the excited state and of the conduction band minimum of the insulating layer, the localization of the excited states could be very different, in particular their penetration inside the insulating layer.

$\mathrm{NaCl}$ is an insulating ionic crystal with large forbidden band gap of $8.5 \mathrm{eV}$ and a conduction band minimum (CBM) located at $0.6 \mathrm{eV}$ below the vacuum level. ${ }^{16,17}$ Potentially, it can thus be very efficient as a decoupling surface coating. The presence of ionic charges inside the layer makes it very different from a condensed rare gas, and that makes it an appealing system to study from fundamental and practical points of view. Adsorption of atomically thin $\mathrm{NaCl}$ layers has been studied for several metal surfaces (see Refs. 18-21). It was found to retain its ionic structure, the charges influencing the adsorption structure in the case of vicinal surfaces. ${ }^{20,21}$ Even if the position of the CBM can be thought to be different for an adsorbed thin layer as compared to the bulk crystal, $\mathrm{NaCl}$ provides an efficient spacer layer for excited states not too close to the vacuum level. This has been proven by recent scanning tunneling microscopy (STM) experiments on several systems. For pentacene molecules adsorbed on ultrathin $\mathrm{NaCl}$ layers on $\mathrm{Cu}(111)^{18,22}$ a broad band gap overlapping the system Fermi level was observed, as well as an efficient decoupling of the pentacene molecular orbitals from the substrate due to the $\mathrm{NaCl}$ coating. It has also been demonstrated that individual gold adatoms on an ultrathin $\mathrm{NaCl}$ film supported by a copper surface exhibit two different stable charge states (neutral and negative), which can be controlled by adding or removing a single electron to or from the adatom with a scanning tunneling microscope tip. Here, the negative Au ion does not decay by electron tunneling into the metal substrate specifically because of the decoupling effect of the $\mathrm{NaCl}$ spacer layer. ${ }^{23,24}$ Similarly, stable neutral, positive, and negative ion states have been reported for the individual silver adatoms on the $\mathrm{NaCl} / \mathrm{Cu}$ surface. $^{25,26}$

On a clean $\mathrm{Cu}(111)$ surface, an image potential state, labeled $n=1$, is present. It corresponds to an electron attracted in the image potential of the surface and unable to penetrate inside bulk $\mathrm{Cu}$ because of the surface-projected band gap of $\mathrm{Cu}(111)$ (see Refs. 27-29). This state is stable in a one-electron picture and decays via inelastic interactions with the bulk electrons. In principle, image potential states form a Rydberg 
series of states bound in the image potential in front of the surface. However, on a clean $\mathrm{Cu}(111)$ surface, higher lying excited states $(n>1)$ are actually resonances because their energy is above the top of the surface-projected band gap of $\mathrm{Cu}(111)$ and they can also decay by one-electron transfer into the bulk, leading to shorter lifetimes. When an ultrathin insulating $\mathrm{NaCl}$ layer is adsorbed on the $\mathrm{Cu}(111)$ surface, the question arises of the fate of the ISs (states and resonances) of the clean surface: Are they repelled into vacuum, or do they mix with conduction band states or with interface states? Recently, TR-2PPE studies of excited states on a $\mathrm{Cu}(111)$ surface covered with several atomic layers of $\mathrm{NaCl}^{30}$ revealed the existence of two excited states with a lifetime of a few hundred fs located a fraction of an $\mathrm{eV}$ below the vacuum level. Although these states are located in the energy range where ISs are expected, they were not assigned to ISs but to the $\mathrm{NaCl}$ layer conduction band and to a surface state split off the conduction band. ${ }^{30}$ In addition, the energies of these states were seen to vary with time after their formation, and this was interpreted as a sign of the transformation of the photoexcited states into two-dimensional (2D) small polarons. In the present paper, we report on a theoretical study of the excited states in the $\mathrm{NaCl} / \mathrm{Cu}(111)$ system. The aim is to compute the excited-state properties (energy, lifetime, and associated wave function) and to characterize the role of an ultrathin $\mathrm{NaCl}$ layer in perturbing the excited states located at the $\mathrm{Cu}(111)$ surface. We are thus able to assign the states and, in particular, to specify their spatial location with respect to the insulating layer. We also discuss the link between wave-function properties, state lifetime, and the state's possible evolution into a small polaron.

The paper is organized as follows. The next section explains the theoretical methods employed. Our results are shown in Sec. III. Our discussion and conclusions are in Sec. IV. Throughout the paper, atomic units are used, unless otherwise stated.

\section{METHOD AND COMPUTATIONAL DETAILS}

The present theoretical study is based on a time-domain wave packet propagation (WPP) treatment of the dynamics of an excited electron moving in a model potential. We determined the energies, lifetimes, and wave functions of the excited electronic states at the surface of $\mathrm{NaCl}$ ultrathin films deposited on $\mathrm{Cu}(111)$. For the sake of comparison with available experimental data, systems with 1, 2, 3, and 4 monolayers (ML) of $\mathrm{NaCl}$ on $\mathrm{Cu}(111)$ have been considered.
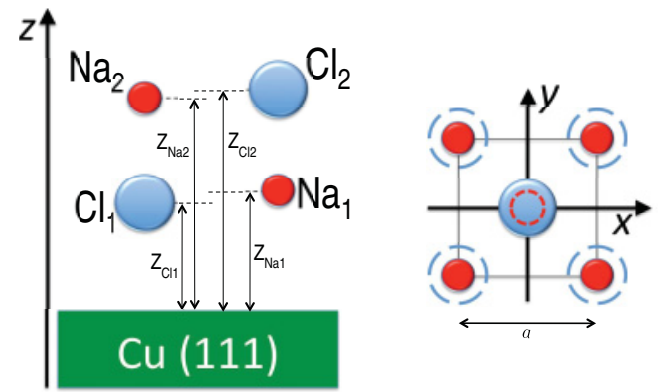

FIG. 1. (Color online) Schematic representation of the 2ML$\mathrm{NaCl} / \mathrm{Cu}(111)$ system. In the left panel a view in a plane perpendicular to the surface is shown (the $z$ axis is normal to the surface). In the right panel the unit cell used for the WPP calculations in the surface plane $(x y)$ is shown; the arrow shows the unit cell side $a=\frac{l}{\sqrt{2}}=$ $3.8679 \AA$ ( $l=$ lattice constant). Small red circles, Na atoms; big blue circles, $\mathrm{Cl}$ atoms.

The general idea of our approach is to study by WPP the time evolution of an excited electron evolving in a local model potential describing the interaction between an additional electron and the $\mathrm{NaCl} / \mathrm{Cu}(111)$ system. An analysis of the time dependence of the electron wave packet yields all the required information about the quasistationary electronic states in the system.

\section{A. Geometry}

Figure 1 sketches the system with $2 \mathrm{ML}$ of $\mathrm{NaCl}$ on a $\mathrm{Cu}(111)$ surface. Bennewitz et al. and Repp et al. (see, e.g., Refs. 18 and 31) have shown that $\mathrm{NaCl}$ grows on a $\mathrm{Cu}(111)$ surface as an incommensurate square lattice, leading to moiré patterns in STM experiments. ${ }^{18} \mathrm{~A}$ similar adsorption geometry with a moiré pattern was also reported on a $\operatorname{Ag}(100)$ susbstrate. ${ }^{32}$ At room temperature, growth starts with doublelayer islands with carpet-like covering of defects. ${ }^{18}$ In our model study, we have taken the geometry of the adsorbed layers from previous theoretical calculations ${ }^{33}$ and experimental measurements. ${ }^{31}$ The lattice constant of the two-dimensional $\mathrm{NaCl}$ layer, $l=5.47 \AA$, has been taken from the LEED experimental results of Ref. 31. The adsorption heights used here for the $\mathrm{Na}^{+}\left(Z_{\mathrm{Na}}\right)$ and $\mathrm{Cl}^{-}\left(Z_{\mathrm{Cl}}\right)$ ions in the successive layers are given in Table I for the $\mathrm{NaCl}$ films of different thickness. As the main feature, $Z_{\mathrm{Na}}$ and $Z_{\mathrm{Cl}}$ are different in the first layer and the difference rapidly decreases for layers farther away from the surface. For 1 and $2 \mathrm{ML} \mathrm{NaCl}$ films, we used the results of the $a b$ initio study performed for the $\mathrm{NaCl} / \mathrm{Cu}(100)$ system. ${ }^{33}$ The $Z_{\mathrm{Na}}$ and $Z_{\mathrm{Cl}}$ values calculated by Olsson et al. ${ }^{33}$ for various adsorption sites in each layer

TABLE I. Distance in $\AA$ between the ions $\left(\mathrm{Na}^{+}\right.$or $\left.\mathrm{Cl}^{-}\right)$and the surface layer of $\mathrm{Cu}(111)$ atoms in the different systems studied.

\begin{tabular}{|c|c|c|c|c|c|c|c|c|}
\hline & \multicolumn{2}{|c|}{ 1st Layer } & \multicolumn{2}{|c|}{ 2nd Layer } & \multicolumn{2}{|c|}{ 3rd Layer } & \multicolumn{2}{|c|}{ 4th Layer } \\
\hline & $\mathrm{Cl}^{-}$ & $\mathrm{Na}^{+}$ & $\mathrm{Cl}^{-}$ & $\mathrm{Na}^{+}$ & $\mathrm{Cl}^{-}$ & $\mathrm{Na}^{+}$ & $\mathrm{Cl}^{-}$ & $\mathrm{Na}^{+}$ \\
\hline $1 \mathrm{ML}$ & 3.1475 & 3.0100 & & & & & & \\
\hline $2 \mathrm{ML}$ & 3.1450 & 3.2325 & 6.1300 & 6.0300 & & & & \\
\hline $3 \mathrm{ML}$ & 3.1450 & 3.2325 & 6.1300 & 6.0300 & 8.97125 & 8.97125 & & \\
\hline $4 \mathrm{ML}$ & 3.1450 & 3.2325 & 6.1300 & 6.0300 & 8.97125 & 8.97125 & 11.8625 & 11.8625 \\
\hline
\end{tabular}


have been averaged. This procedure removes the corrugation in the layer due to the incommensurate growth, but retains the difference in adsorption height between the $\mathrm{Na}^{+}$and $\mathrm{Cl}^{-}$ions in each layer. For the $3 \mathrm{ML}$ coverage, the only available $a b$ initio data correspond to the $3 \mathrm{ML}-\mathrm{NaCl} / \mathrm{Cu}(311)$ case. ${ }^{34}$ It has been demonstrated that (i) the adsorption height difference between $\mathrm{Na}^{+}$and $\mathrm{Cl}^{-}$in the third layer was very small, and (ii) the distance between the third and second layers was very close to that between the first and second layers. Based on these results, $Z_{\mathrm{Na}}=Z_{\mathrm{Cl}}$ has been used here for the third layer with the third-to-second interlayer distance equal to that between the second and first layers. Finally, the fourth layer was generated in the same way $\left(Z_{\mathrm{Na}}=Z_{\mathrm{Cl}}\right.$ and the same interlayer distance).

In what follows we define the electron-surface coordinate $z$ with respect to the image potential plane of the substrate, which is located 2.11 a.u. in front of the surface plane of $\mathrm{Cu}$ atoms. ${ }^{35}$ The origin of the $x$ and $y$ coordinates is on a $\mathrm{Cl}^{-}$ion of the first layer (see Fig. 1).

\section{B. Wave packet propagation}

The WPP approach consists in solving the time-dependent Schrödinger equation for the wave function of the excited electron $\Psi(\mathbf{r}, t)$. The details of the method and its application for excited states at surfaces can be found in Refs. 29 and 36. We thus only discuss the aspects specific to the present study.

With the present modeling (see below), the $\mathrm{NaCl} / \mathrm{Cu}(111)$ system has the same translational symmetry as the $\mathrm{NaCl}$ layer: it is periodic in the $x$ and $y$ coordinates parallel to the surface with a square $a \times a$ unit cell (see Fig. 1). The wave function $\Psi(\mathbf{r}, t)$ is then written as a Bloch wave in Cartesian coordinates $\mathbf{r}=(x, y, z)$ :

$$
\Psi(\mathbf{r}, t)=e^{i k_{x} x} e^{i k_{y} y} \psi_{k_{x} k_{y}}(\mathbf{r}, t),
$$

where $k_{x}$ and $k_{y}$ are the components of the electron wave vector parallel to the surface. $\psi_{k_{x} k_{y}}(\mathbf{r}, t)$ is a periodic function of the $x$ and $y$ coordinates. Given an initial condition $\psi_{k_{x} k_{y}}(\mathbf{r}, t=0)=$ $\psi_{0}(\mathbf{r})$, the time evolution of $\psi_{k_{x} k_{y}}(\mathbf{r}, t)$ is calculated with the short-time propagation scheme:

$$
\psi_{k_{x} k_{y}}(\mathbf{r}, t+\Delta t)=e^{-i H_{k_{x} k_{y}} \Delta t} \psi_{k_{x} k_{y}}(\mathbf{r}, t) .
$$

The "effective" Hamiltonian $H_{k_{x} k_{y}}$ in Eq. (2) is given by

$$
\begin{aligned}
H_{k_{x} k_{y}}= & -\frac{1}{2}\left[\left(\frac{\partial}{\partial x}+i k_{x}\right)^{2}+\left(\frac{\partial}{\partial y}+i k_{y}\right)^{2}+\frac{\partial^{2}}{\partial z^{2}}\right] \\
& +V(x, y, z),
\end{aligned}
$$

where the term in square brackets is the kinetic energy operator and $V(x, y, z)$ is the total potential of the system.

The time propagation given by Eq. (2) is performed numerically using the split-operator technique for the exponential of the Hamiltonian operator ${ }^{37}$ and the Fourier-grid pseudospectral approach for the kinetic energy term. ${ }^{38}$ A time propagation step $\Delta t=0.1$ a.u. was used. The wave function $\psi_{k_{x} k_{y}}(\mathbf{r}, t)$ was represented on the equidistant mesh in $x, y$, and $z$ coordinates with $\left(N_{x}=64\right) \times\left(N_{y}=64\right) \times\left(N_{z}=3072\right)$ points and the spatial step $\Delta x=\Delta y=\Delta z=a / N_{x} .{ }^{39}$ The large number of $z$ points is mandatory to span a distance range extending from -165.0 a.u. inside the metal to 185.7 a.u. into the vacuum side, so that several of the extended excited states close to the vacuum level of the system are well reproduced.

The characteristics (energies and lifetimes) of the excited electronic states of the system are obtained from the analysis of the survival amplitude:

$$
A(t)=\left\langle\psi_{0}(\mathbf{r}) \mid \psi_{k_{x} k_{y}}(\mathbf{r}, t)\right\rangle,
$$

as explained in Refs. 29 and 36. Moreover, the WPP allows the extraction of the wave function of the corresponding quasistationary state at the energy $E$ :

$$
\Psi(E, \mathbf{r})=\int_{0}^{\infty} e^{i E t} \Psi(\mathbf{r}, t) d t .
$$

The information on $\Psi_{E}(\mathbf{r})$ is extremely helpful for the analysis of the results, in particular for the assignment of the different quasistationary excited states of the system as discussed below.

The total potential $V(x, y, z)$ [see Eq. (3)] acting on an excited electron is given by the sum of several (Hermitian and non-Hermitian) contributions:

$$
V(x, y, z)=V_{\text {Model }}(x, y, z)+V_{\mathrm{ee}}(z)+V_{\text {Opt }}(z) .
$$

$V_{\text {Model }}(x, y, z)$ is the model electrostatic potential describing electron interaction with the $\mathrm{Cu}(111)$ surface coated with adsorbate layers of $\mathrm{NaCl}$. The detailed discussion on $V_{\text {Model }}(x, y, z)$ is presented below.

$V_{\text {ee }}(z)$ is the imaginary potential introduced to account for the many-body contribution to the excited-state decay. Indeed, the WPP approach describes a one-electron dynamics and so it addresses the decay of the excited states at surfaces by oneelectron energy-conserving transitions into the continuum of bulk states of the substrate. The inelastic electron-electron scattering inside the metal substrate also leads to the decay of the excited states via energy relaxation. The inelastic decay can be described with an additional many-body treatment, ${ }^{40}$ or by inclusion of a non-Hermitian (absorbing) potential into the WPP scheme as, e.g., in Ref. 13. The inclusion of an absorbing potential $V_{\text {ee }}(z)$ follows approaches developed in low-energy electron diffraction studies ${ }^{41}$ and is much linked to the computation of the image potential state lifetimes in terms of penetration factors (see discussions in Refs. 42 and 43).

In the present work, we used $V_{\mathrm{ee}}(z)$ of the form

$$
V_{\mathrm{ee}}(z)= \begin{cases}-i\left(\frac{1}{2} e^{-z}\right) \chi_{\mathrm{ee}} & \text { for } \quad z>0 \\ -i\left(\frac{1}{1+e^{z}}\right) \chi_{\mathrm{ee}} & \text { for } \quad z<0\end{cases}
$$

The parameter $\chi_{\mathrm{ee}}=0.0535 \mathrm{eV}$ has been set in such a way that the experimental width of the first image potential state in clean $\mathrm{Cu}(111)\left(\Gamma=30 \pm 10 \mathrm{meV}^{44}\right)$ is well reproduced. $V_{\text {ee }}$ is shown in Fig. 2. Performing calculations with and without this term allows us to determine both elastic and inelastic (one-electron and multielectron) contributions to the decay of the excited states.

Finally $V_{\text {Opt }}(z)$ is an absorbing potential ${ }^{45}$ introduced at the edge of the $z$ mesh inside the metal to suppress the artificial reflections of the wave packet. The outgoing wave boundary conditions required to determine the quasistationary states are therefore enforced. 


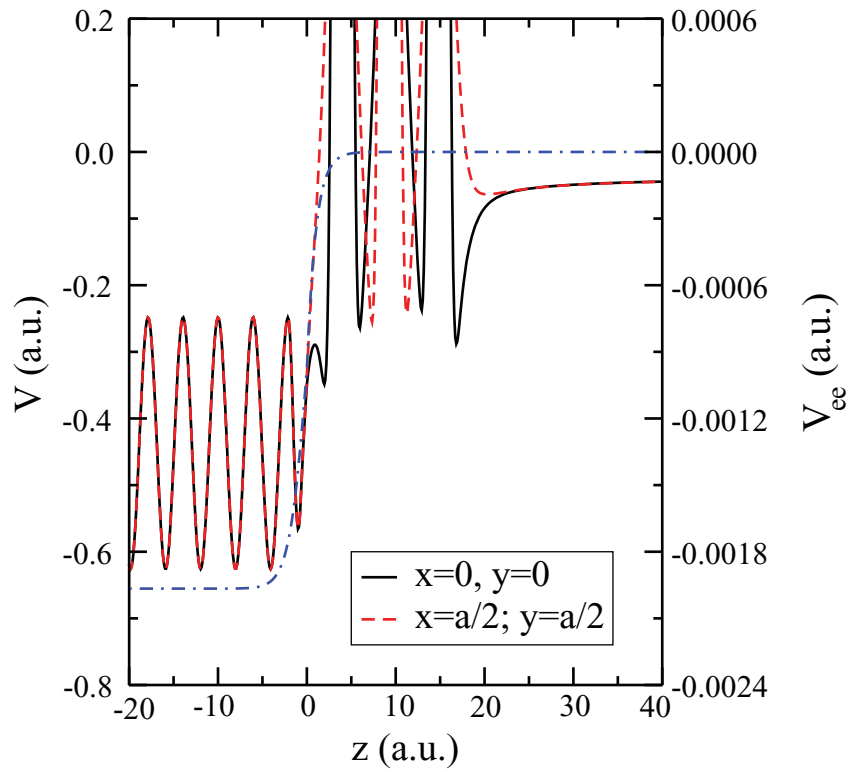

FIG. 2. (Color online) Computed potential (in a.u.) describing the interaction of the electron with the $3 \mathrm{ML}-\mathrm{NaCl} / \mathrm{Cu}(111)$ system. The potential is plotted as a function of the coordinate perpendicular to the surface $z$. The black full line shows the potential along the $x=y=0$ line, which contains the center of a $\mathrm{Cl}$ atom in the first and third layers and a $\mathrm{Na}$ atom in the second layer. The red dashed line shows the potential along the $x=y=a / 2$ line, which contains the center of a $\mathrm{Na}$ atom in the first and third layers and a $\mathrm{Cl}$ atom in the second layer. The blue dash-dotted line shows the imaginary part of the $V_{\text {ee }}$ potential [Eq. (7)].

\section{Model electrostatic potential}

The model electrostatic potential $V_{\text {Model }}(x, y, z)=$ $V_{\mathrm{NaCl}}(x, y, z)+V_{\text {Surf }}(z)$ describing the electron interaction with the $\mathrm{Cu}(111)$ surface coated with adsorbate layers of $\mathrm{NaCl}$ is defined as the sum of two terms. $V_{\text {Surf }}(z)$ is the electron interaction with a clean metal surface, and $V_{\mathrm{NaCl}}(x, y, z)$ is the change in the electron-surface interaction due to the presence of $\mathrm{NaCl}$ coating. This modeling is very similar to the one used in our earlier description of $\mathrm{Ar}$ layers on $\mathrm{Cu}(100)^{14}$ and of $\mathrm{Cu}$ adatoms and $\mathrm{Cu}$ wires on $\mathrm{Cu}$ surfaces. ${ }^{46,47}$ It has the advantage of being well adapted to describe the perturbation of the surface excited states and, in particular, image potential states by the adsorbate layers.

The starting point for the calculation of the $\mathrm{V}_{\mathrm{NaCl}}$ potential induced by the $\mathrm{NaCl}$ layer is to set the binary electron interactions with $\mathrm{Na}^{+}$and $\mathrm{Cl}^{-}$ions forming the ionic crystal lattice: $V_{\mathrm{Na}}$ and $V_{\mathrm{Cl}}$.

For the electron- $\mathrm{Na}^{+}$interaction, we have taken the pseudopotential proposed by Bardsley: ${ }^{48}$

$$
\begin{aligned}
V_{\mathrm{Na}}(R)= & -\frac{1}{R}-\frac{\alpha_{d}^{\mathrm{Na}}}{2\left(R^{2}+a_{\mathrm{Na}}^{2}\right)^{2}}-\frac{\alpha_{q}^{\mathrm{Na}}}{2\left(R^{2}+a_{\mathrm{Na}}^{2}\right)^{3}} \\
& +A_{\mathrm{Na}} \exp \left[-\gamma_{\mathrm{Na}} R^{2}\right],
\end{aligned}
$$

where $R$ is the electron-ion distance. The first term is the Coulomb attraction by the positive ion core, the second and third terms correspond to the dipole and quadrupole interactions, respectively, and the last term corresponds to the repulsive short-range part of the potential. The original Bardsley pseudopotential ${ }^{48}$ depends on the electron momentum $\ell$. Here we assume that the low-energy part of the $\mathrm{NaCl}$ conduction band (important in the present study) is dominated by the $s$ wave so that only the $\ell=0$ potential is retained. With this choice the values for the different parameters are ${ }^{48}$ dipole polarizability $\alpha_{d}^{\mathrm{Na}}=0.945$ a.u., quadrupole polarizability $\alpha_{q}^{\mathrm{Na}}=5.0$ a.u., cutoff factor $a_{\mathrm{Na}}^{2}=1.21$ a.u., amplitude $A_{\mathrm{Na}}=10.28159$ a.u., and $\gamma_{\mathrm{Na}}=1.294506$ a.u.

The electron- $\mathrm{Cl}^{-}$interaction is defined along the same lines:

$$
V_{\mathrm{Cl}}(R)=+\frac{1}{R}-\frac{\alpha_{d}^{\mathrm{Cl}}}{2\left(R^{2}+a_{\mathrm{Cl}}^{2}\right)^{2}}+A_{\mathrm{Cl}} \exp \left[-R^{2} / a_{\mathrm{Cl}}^{2}\right] .
$$

The values of the different parameters have been set as follows: The dipole polarizability $\alpha_{d}^{\mathrm{Cl}}=20.65$ a.u. has been taken from Refs. 49 and 50; the cutoff factor $a_{\mathrm{Cl}}=1.9326$ a.u. has been computed as the mean value of $R$ from the radial part of the $p$ orbital of $\mathrm{Cl}^{-}$from Ref. 51. The amplitude of the short-range repulsive part $A_{\mathrm{Cl}}=0.68$ a.u. has been set in such a way that the WPP calculation for the bulk $\mathrm{NaCl}$ crystal reproduces the experimentally measured energy of the conduction band bottom equal to $-0.6 \mathrm{eV}$ with respect to the vacuum level. ${ }^{16,17}$

Using the binary interaction potentials defined by Eqs. (8) and (9), the potential induced by the $\mathrm{NaCl}$ layer is sought in the form

$$
V_{\mathrm{NaCl}}(x, y, z)=V_{\mathrm{C}}(x, y, z)+V_{\mathrm{Pol}}(x, y, z)+V_{\mathrm{SR}}(x, y, z),
$$

where $V_{\mathrm{C}}$ denotes the Coulomb potential, $V_{\mathrm{Pol}}$ the polarization potential, and $V_{\mathrm{SR}}$ the short-range potential.

The short-range potential $V_{\mathrm{SR}}$ is defined as the sum of the short-range potentials due to the $\mathrm{Na}^{+}$and $\mathrm{Cl}^{-}$ions in the ionic crystal film:

$$
\begin{aligned}
V_{\mathrm{SR}}(x, y, z)= & \sum_{\mathrm{Na}_{i}}\left\{-\frac{\alpha_{q}^{\mathrm{Na}}}{2\left(\left|\mathbf{R}_{i}-\mathbf{r}\right|^{2}+a_{\mathrm{Na}}^{2}\right)^{3}}\right. \\
& \left.+A_{\mathrm{Na}} \exp \left[-\gamma_{\mathrm{Na}}\left|\mathbf{R}_{i}-\mathbf{r}\right|^{2}\right]\right\} \\
& +A_{\mathrm{Cl}} \sum_{\mathrm{Cl}_{j}} \exp \left[-\left|\mathbf{R}_{j}-\mathbf{r}\right|^{2} / a_{\mathrm{Cl}}^{2}\right] .
\end{aligned}
$$

The first sum runs over the $\mathrm{Na}^{+}$ionic sites located at $\mathbf{R}_{i}=$ $\left(X_{i}, Y_{i}, Z_{i}\right)$, and the second sum runs over the $\mathrm{Cl}^{-}$ionic crystal sites located at $\mathbf{R}_{j}=\left(X_{j}, Y_{j}, Z_{j}\right)$. We recall that $\mathbf{r}=(x, y, z)$ stands for the electron coordinate. The summations converge quickly for this short-range part of the potential. Typically $98 \mathrm{Cl}^{-}$and $98 \mathrm{Na}^{+}$ions are required.

The Coulomb potential $V_{C}$ accounts for the Coulomb interaction of the active electron with $\mathrm{Na}^{+}$and $\mathrm{Cl}^{-}$ions in the successive layers forming the $\mathrm{NaCl}$ film, as well as with their classical electrostatic images formed by the metal surface:

$$
\begin{gathered}
V_{C}(x, y, z \geqslant 0)=\sum_{n} Q_{n}\left\{-\frac{1}{\left|\mathbf{R}_{n}-\mathbf{r}\right|}+\frac{1}{\left|\mathbf{R}_{n}^{\prime}-\mathbf{r}\right|}\right\}, \\
V_{C}(x, y, z<0)=0,
\end{gathered}
$$

where the summation runs over the ionic sites of the $\mathrm{NaCl}$ film. $Q_{n}$ is the charge of the ion located at the $n$th site 
$(+1$ for $\mathrm{Na}$ and -1 for $\mathrm{Cl}) ; \mathbf{R}_{n}=\left(X_{n}, Y_{n}, Z_{n}\right)$ and $\mathbf{R}_{n}^{\prime}=$ $\left(X_{n}, Y_{n},-Z_{n}\right)$ are the coordinates of the ion at the $n$th site and its electrostatic image (the image plane being the origin of the $z$ coordinate). Complete screening of the potential is assumed inside the metal. In practice, for the electron close to the $\mathrm{NaCl}$ film, $V_{C}(x, y, z)$ is calculated directly from Eq. (12). The ionic crystal lattice is built by adding successive groups of positive and negative ions forming high-order multipoles, which ensures the total charge neutrality and fast convergence. For an electron far from the surface, we use the solution of the periodic Poisson equation in the reciprocal space. Thus, the potential created by the periodic 2D arrangement of charges $Q$ reads

$$
\begin{aligned}
V_{Q}(x, y, z)= & -\frac{2 \pi Q}{S}(|z-Z| \\
& \left.-\sum_{\ell, j} \frac{1}{G_{\ell j}} e^{-G_{\ell j}|z-Z|} e^{\left.i G_{\ell}(x-X)+G_{j}(y-Y)\right]}\right) .
\end{aligned}
$$

The $(X, Y, Z)$ are the coordinates of the charge $Q$ in the unit cell. $S=a^{2}$ is the area of the unit cell. With the $\hat{e}_{x}\left(\hat{e}_{y}\right)$ unit length vector in the $x(y)$ direction, the reciprocal vectors of the $2 \mathrm{D}$ lattice are given by

$$
\mathbf{G}_{\ell j}=\hat{e}_{x} G_{\ell}+\hat{e}_{y} G_{j}=\frac{2 \pi}{a}\left(\hat{e}_{x} \ell+\hat{e}_{y} j\right),
$$

$G_{\ell j}=\frac{2 \pi}{a} \sqrt{\ell^{2}+j^{2}}$. The summation in Eq. (13) runs over $\ell$ and $j$ such that $G_{\ell j} \neq 0$. Each layer forming $\mathrm{NaCl}$ film contributes to the $V_{C}$ potential with four terms of the type given by Eq. (13): one for the $\mathrm{Na}^{+}$ions, one for the $\mathrm{Cl}^{-}$ions, one for electrostatic images of the $\mathrm{Na}^{+}$ions, and one for electrostatic images of the $\mathrm{Cl}^{-}$ions.

The polarization potential $V_{\mathrm{Pol}}(x, y, z)$ describes the interaction of the excited electron with electric dipoles $\mathbf{P}_{n}$ induced at ionic sites of the $\mathrm{NaCl}$ film, as well as with electrostatic images of the dipoles $\mathbf{P}_{n}^{\prime}$. It has to be calculated self-consistently in order to account for the dielectric properties of the $\mathrm{NaCl}$. The dipole induced at one ionic site contributes to the electric field and thus to the dipoles at other sites, leading to the mutual depolarization and screening of the applied field.

Thus, the field created at the given ionic site $m$ is given by

$$
\begin{aligned}
\mathbf{E}_{m}(\mathbf{r})= & -\frac{\mathbf{R}_{m}-\mathbf{r}}{\left|\mathbf{R}_{m}-\mathbf{r}\right|^{3}}+\frac{\mathbf{R}_{m}-\mathbf{r}^{\prime}}{\left|\mathbf{R}_{m}-\mathbf{r}^{\prime}\right|^{3}} \\
& +\sum_{n \neq m} Q_{n}\left\{\frac{\mathbf{R}_{m n}}{\left|\mathbf{R}_{m n}\right|^{3}}-\frac{\mathbf{R}_{m n}^{\prime}}{\left|\mathbf{R}_{m n}^{\prime}\right|^{3}}\right\} \\
& +\sum_{n \neq m}\left\{\frac{3 \mathbf{u}_{m n}\left[\mathbf{P}_{n}(\mathbf{r}) \cdot \mathbf{u}_{m n}\right]-\mathbf{P}_{n}(\mathbf{r})}{\left|\mathbf{R}_{m n}\right|^{3}}\right. \\
& \left.+\frac{3 \mathbf{u}_{m n}^{\prime}\left[\mathbf{P}_{n}^{\prime}(\mathbf{r}) \cdot \mathbf{u}_{m n}^{\prime}\right]-\mathbf{P}_{n}^{\prime}(\mathbf{r})}{\left|\mathbf{R}_{m n}^{\prime}\right|^{3}}\right\},
\end{aligned}
$$

where the dependence on the electron coordinates is made explicit. The first two terms stand for the electric field created by an electron and its electrostatic image located at $\mathbf{r}^{\prime}=(x, y,-z)$. The next terms describe the field created by the polarizable ions of the $\mathrm{NaCl}$ film. $\mathbf{R}_{m n}=\mathbf{R}_{m}-\mathbf{R}_{n}$, and $\mathbf{R}_{m n}^{\prime}=\mathbf{R}_{m}-\mathbf{R}_{n}^{\prime}$ with $\mathbf{R}_{n}$ and $\mathbf{R}_{n}^{\prime}$ defined above. $\mathbf{u}_{m n}$ is the unit length vector $\mathbf{u}_{m n}=\mathbf{R}_{m n} /\left|\mathbf{R}_{m n}\right|$; similarly $\mathbf{u}_{m n}^{\prime}=\mathbf{R}_{m n}^{\prime} /\left|\mathbf{R}_{m n}^{\prime}\right|$.
Depending on whether $m$ corresponds to the $\mathrm{Na}^{+}$or $\mathrm{Cl}^{-}$ ionic site of the $\mathrm{NaCl}$ film, the dipole $\mathbf{P}_{m}(\mathbf{r})$ induced by the electric field $\mathbf{E}_{m}(\mathbf{r})$ is given by

$$
\mathbf{P}_{m}(\mathbf{r})=\frac{\alpha_{d}^{\mathrm{Na} / \mathrm{Cl}}}{\left[1+a_{\mathrm{Na} / \mathrm{Cl}}^{2}\left|\mathbf{E}_{m}(\mathbf{r})\right|\right]^{3}} \mathbf{E}_{m}(\mathbf{r}) .
$$

The saturation of the linear relation between the field the ion dipole corresponds to the saturation of the polarization part of the potential in Eqs. (8) and (9).

Equations (15) and (16) are solved iteratively until the desired level of convergence is reached for the dipoles $\mathbf{P}_{m}(x, y, z)$. The polarization potential $V_{\mathrm{Pol}}(x, y, z)$ is then obtained from the self-consistent field $\mathbf{E}_{\mathrm{SC}}$ created by the dipoles and their images:

$$
V_{\mathrm{Pol}}(x, y, z)=\int_{\infty}^{z} \hat{e}_{z} \mathbf{E}_{\mathrm{SC}}(x, y, \xi) d \xi
$$

$\hat{e}_{z}$ is the unit length vector in the positive direction of the $z$ axis. The procedure described here has been already used earlier in the case of rare-gas layers in Ref. 14. We have found that the convergence can be greatly improved if one first calculates the dipoles induced at $\mathrm{NaCl}$ lattice sites by the field of the ionic film, the active electron being absent. From the periodicity of the system it follows that within each sublayer forming $\mathrm{NaCl}$ film, the dipoles induced at $\mathrm{Na}^{+}$sites are equal (the same for the $\mathrm{Cl}^{-}$sites). Moreover, for symmetry reasons the permanent dipoles are along the $z$ axis. In this case, similarly to Eq. (13) one can use the reciprocal vector expansion of the potential created by the periodic layer of the dipoles $\mathbf{P}=\hat{e}_{z} P$ for the fast calculation of the potential at a large distance $z$ from the surface:

$$
V_{\uparrow}=-\frac{2 \pi}{S} P\left(1+\sum_{\ell, j}^{\ell \neq 0 ; j \neq 0} e^{-G_{\ell j}|z-Z|} e^{i\left[G_{\ell}(x-X)+G_{j}(y-Y)\right]}\right),
$$

where the same notations as in Eq. (13) are used. In the second step, the changes of the dipoles due to the external electron are calculated.

While the 3D structure of the $\mathrm{NaCl}$ monolayer is explicitly introduced in the present study via the $V_{\mathrm{NaCl}}(x, y, z)$ potential, the interaction of an excited electron with the clean $\mathrm{Cu}(111)$ surface is modeled by a one-dimensional potential $V_{\text {Surf }}(z)$, a function of the electron coordinate perpendicular to the surface $z$ only. Our model is based on the potential that has been introduced by Chulkov et al. ${ }^{35}$ for studies of the energies and lifetimes of the surface and image potential states. This potential has been designed in such a way that the main features of the electronic structure of the $\mathrm{Cu}(111)$ surface are correctly reproduced at the $\bar{\Gamma}$ point: the projected band gap between $-5.83 \mathrm{eV}$ and $-0.69 \mathrm{eV}$, the Shockley-type surface state at $-5.27 \mathrm{eV}$, and the first image potential state at $-0.82 \mathrm{eV}$ (all energies with respect to the vacuum level). The oscillating structure of the potential inside the metal reflects the periodic arrangement of the successive $\mathrm{Cu}(111)$ planes. A free-electron motion parallel to the surface is assumed. Importantly, the image potential tail of the electron-surface interaction is explicitly introduced for an electron in the 
TABLE II. Calculated electrostatic contribution (Elect) and measured (exp) work function change $(\Delta \Phi)$ in $\mathrm{eV}$ from Ref. 52.

\begin{tabular}{lrr}
\hline \hline & $\Delta \Phi_{\text {Elect }}$ & $\Delta \Phi_{\text {exp }}$ \\
\hline $1 \mathrm{ML}$ & 0.575 & -1.0 \\
$2 \mathrm{ML}$ & -0.158 & -1.0 \\
$3 \mathrm{ML}$ & -0.114 & -1.0 \\
$4 \mathrm{ML}$ & -0.111 & -1.0 \\
\hline \hline
\end{tabular}

vacuum. This is a necessary condition for the present study addressing the dynamics of excited states close to the vacuum level. The specificity of the present system, however, precludes the direct use of the original potential proposed in Ref. 35. Indeed, as confirmed by the experiment, the $\mathrm{NaCl}$ coating changes the work function of the surface $\Phi$. The work function change $\Delta \Phi_{\exp }$ has to be correctly reproduced by the potential $V_{\text {Model }}(x, y, z)=V_{\mathrm{NaCl}}(x, y, z)+V_{\text {Surf }}(z)$ since it determines the energy position of the excited electronic states with respect to the projected electronic structure of the $\mathrm{Cu}(111)$ substrate.

Our calculation of the $V_{\mathrm{NaCl}}(x, y, z)$ potential contains the work function change due to the pure electrostatic effect. The difference in heights of the $\mathrm{Na}^{+}$and $\mathrm{Cl}^{-}$ions within each layer in the $\mathrm{NaCl}$ film together with the dipoles that this difference induces at $\mathrm{Na}^{+}$and $\mathrm{Cl}^{-}$ionic crystal sites results in an effective dipole layer that changes the work function of the surface. Table II summarizes the electrostatic contribution to the work function change as calculated here, $\Delta \Phi_{\text {Elect }}$, and as measured experimentally, $\Delta \Phi_{\text {exp }}$, for different $\mathrm{NaCl}$ coverages.

The difference between the calculated electrostatic contribution and measured data is tentatively attributed to the effect of the compression of the electron density on the metal surface by the adsorbate layer (see the discussion in Ref. 53). Our pure electrostatic treatment of $V_{\mathrm{NaCl}}$ does not account for this phenomenon. The $V_{\text {Surf }}(z)$ is then defined as the model potential of Ref. $35, V_{\mathrm{Cu}(111)}$, modified in such a way that the total potential $V_{\text {Model }}=V_{\mathrm{NaCl}}+V_{\text {Surf }}$ reproduces the experimentally measured work function. In order to ensure the correct energy of the projected electronic structure of the $\mathrm{Cu}(111)$ substrate with respect to the vacuum level, the $A_{10}=-11.895 \mathrm{eV}$ parameter of the original potential as given in Ref. 35 has been set to $A_{10}=-11.895 \mathrm{eV}-\Delta \Phi_{\exp }+$ $\Delta \Phi_{\text {Elect }}$.

Figure 2 shows the computed $V_{\text {Model }}(x, y, z)=$ $V_{\mathrm{NaCl}}(x, y, z)+V_{\mathrm{Surf}}(z)$ potential for the case of $3 \mathrm{ML}$ $\mathrm{NaCl} / \mathrm{Cu}(111)$. The results are presented as a function of the coordinate perpendicular to the surface and going through $\mathrm{Na}^{+}$and $\mathrm{Cl}^{-}$ions. Figure 3 shows a $2 \mathrm{D}$ projection of the potential in the $x z$ plane, also going through the center of the $\mathrm{Cl}^{-}$and $\mathrm{Na}^{+}$ions. In both figures the potential exhibits an oscillatory behavior inside the metal $(z<0)$ due to the $V_{\text {Surf }}$ potential and at the origin of the surface-projected band gap of the $\mathrm{Cu}(111)$ surface. The repulsive short-range potential part is also quite visible at the center of the ions. One can also notice that there is an attractive potential well around each $\mathrm{Na}^{+}$ion; these wells join through a region of weaker attractive potential between the ions.

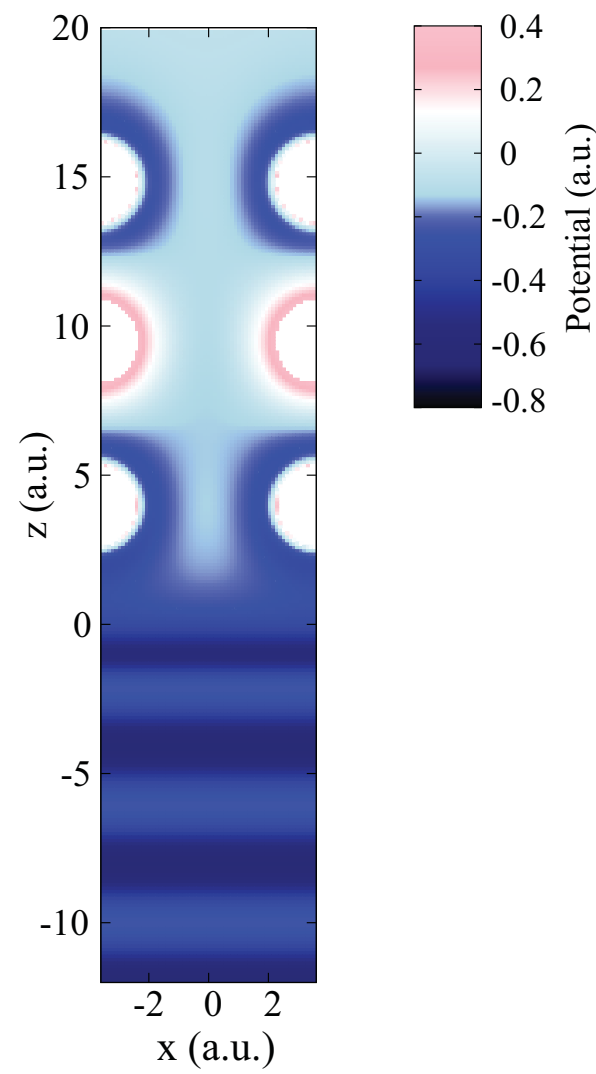

FIG. 3. (Color online) Computed potential (in a.u.) describing the interaction of the electron with the $3 \mathrm{ML}-\mathrm{NaCl} / \mathrm{Cu}(111)$ system. The potential is plotted in the $x z$ plane, as defined in Fig. 1, and relative to the vacuum level; the color code is given in the inset. Note that the potential is plotted in the $y=a / 2$ plane, which contains the centers of $\mathrm{Na}$ atoms in the first and third layers and $\mathrm{Cl}$ atoms in the second layer. The highly repulsive part of the potential (short-range potential) around the center of the atoms is not shown.

\section{RESULTS}

\section{A. Energies and lifetimes}

Several excited states are found in the WPP calculation on the $\mathrm{NaCl} / \mathrm{Cu}(111)$ system. Their energies and lifetimes are shown in Figs. 4 and 5 as functions of the thickness of the $\mathrm{NaCl}$ layer. Other states were also seen at higher energies, most probably corresponding to higher members of the Rydberg series of image potential states. However, these states are very spread out in space in the vacuum region, and our present calculation with a finite computation box in the $z$ direction cannot accurately characterize them. The states in Figs. 4 and 5 correspond to the $\bar{\Gamma}$ point, i.e., to an electron momentum parallel to the surface $k_{\|}=0$. They are labeled $n=1,2$, and 3, following the energy order. Note that this notation merges with the $n$ notation of the image potential states (and resonances) on a clean $\mathrm{Cu}(111)$ surface at zero coverage, although, as is obvious from the following, this does not mean that the states are actual image potential states for finite layer thickness. The energies of the states in Fig. 4 are presented with respect to the Fermi energy $E_{F}$. It appears that the energy of the two higher states roughly follows the variation of the vacuum level 


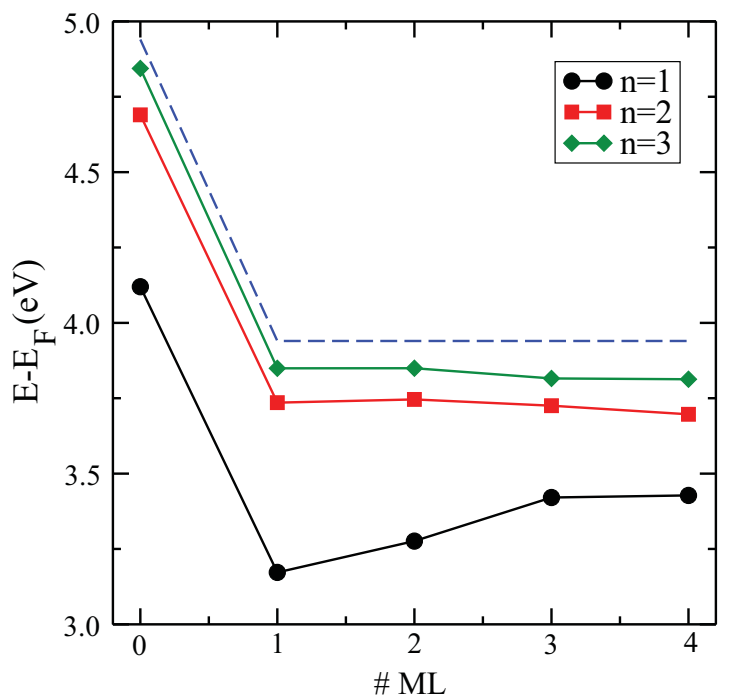

FIG. 4. (Color online) Energy (in eV) with respect to the Fermi level of the $n=1,2$, and 3 resonances in $n \mathrm{ML}-\mathrm{NaCl} / \mathrm{Cu}(111)$ as a function of the number of $\mathrm{NaCl}$ monolayers. Black circles, $n=1$; red squares, $n=2$; green diamonds, $n=3$. The energy of the vacuum level is shown by the blue dashed line.

energy. As for the lowest level, it comes closer to the vacuum level as the thickness increases.

The upper edge of the surface-projected band gap of $\mathrm{Cu}(111)$ is located at $4.26 \mathrm{eV}$ above $E_{F}$. Thus, for all the studied $\mathrm{NaCl}$ coverages, the quasistationary states $n=1$, 2 , and 3, as well as the higher members of the IS series, lie inside the substrate-projected band gap. These $k_{\|}=0$ states cannot decay by energy-conserving one-electron transfer into a $k_{\|}=0$ state of bulk $\mathrm{Cu}$. However, because of the presence of a 2D-periodic $\mathrm{NaCl}$ array on the surface, one

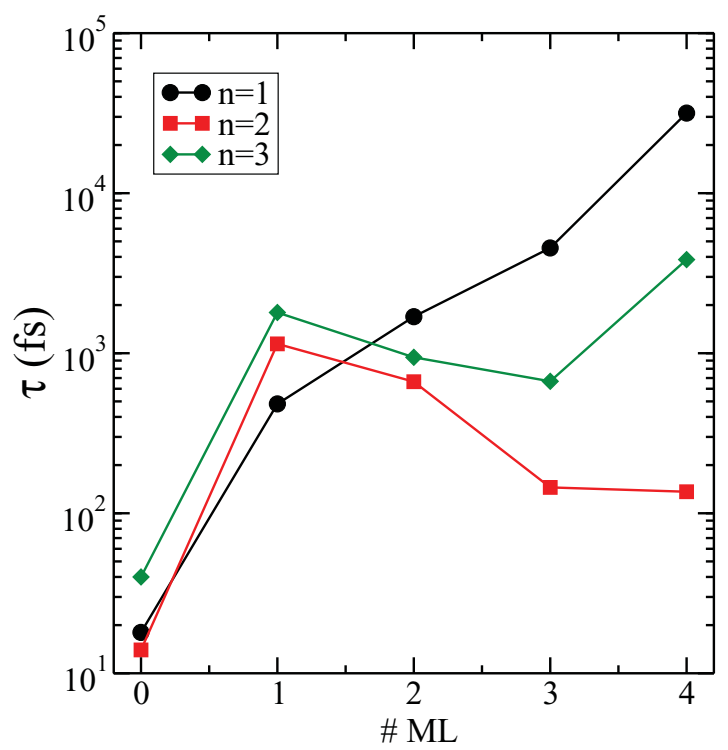

FIG. 5. (Color online) Lifetime (in fs) of the $n=1,2$, and 3 resonances in $n \mathrm{ML}-\mathrm{NaCl} / \mathrm{Cu}(111)$ as a function of the number of $\mathrm{NaCl}$ monolayers. Black circles, $n=1$; red squares, $n=2$; green diamonds, $n=3$. The lifetimes of the image potential states on the clean $\mathrm{Cu}(111)$ surface are taken from Refs. 44 and 59. should also consider transitions assisted by the lattice: the $k_{\|}=0$ quasistationary state decays into bulk states with $\mathbf{k}_{\|}=\mathbf{G}_{\ell j},{ }^{54,55}$ where $\mathbf{G}_{\ell j}$ is one of the reciprocal lattice vectors of the $2 \mathrm{D}-\mathrm{NaCl}$ lattice defined in Eq. (14). In the present study, the $V_{\mathrm{Cu}(111)}(z)$ potential from Ref. 35 assumes a free electron motion parallel to the surface, so that the substrate bands disperse with a free electron mass. Thus, the $n=1-3$ states can decay into $\mathbf{k}_{\|}=\mathbf{G}_{10}$ and $\mathbf{k}_{\|}=\mathbf{G}_{01}$ channels by one-electron transfer assisted by the lattice. However, with the actual effective electron mass in $\mathrm{Cu}(111) m^{*}=0.6$, the lattice-assisted one-electron transfer is forbidden. As a consequence, the $n=1-3$ states only decay by multielectron interactions. We then employed the strategy outlined in Sec. II [calculations with and without the $V_{\mathrm{ee}}(z)$ potential] to evaluate the multielectron decay rate of the quasistationary states. This multielectron rate is the inverse of the state lifetime shown in Fig. 5. The decay rate due to the lattice-assisted one-electron transfer is at most in the few $10^{-4} \mathrm{eV}$ range.

At this point, one can comment on the possibility of lattice-assisted transitions. For energetic reasons, $\mathrm{NaCl}$ latticeassisted transitions are impossible, as well as $\mathrm{Cu}$ latticeassisted transitions [the studied states lie inside the $\mathrm{Cu}(111)$ surface-projected band gap]. However, transitions involving the assistance of both the $\mathrm{NaCl}$ and $\mathrm{Cu}$ lattices are possible. From the localization of the studied states one may expect that the $\mathrm{NaCl}$ lattice shows an influence larger than the $\mathrm{Cu}$ one in the lattice-assisted electron transfer. In the present calculation, the $\mathrm{NaCl}$ lattice-assisted transitions appear as an artifact, although their explicit evaluation shows that their effect is very weak. Therefore, transitions involving reciprocal lattice vector exchange with both $\mathrm{NaCl}$ and $\mathrm{Cu}$ lattices are at least second order, and their effect can be neglected.

The state lifetimes in Fig. 5 exhibit a nonmonotonic variation with the $\mathrm{NaCl}$ layer thickness. This is not the behavior expected for ISs repelled in vacuum by the presence of a thin insulating layer. In this case, since the state is repelled into a region of weaker image potential, the binding energy of the ISs with respect to vacuum decreases when the layer thickness increases. In addition, the probability of the presence of the IS electron inside the bulk decreases and so does the multielectron contribution to the state decay. Actually, the lifetimes are expected to increase rapidly with the thickness, in a roughly exponential variation with the number of layers. ${ }^{6-9,15}$ One also expects the lifetime of the ISs to increase as $n^{3}$ with the index $n$ of the states. ${ }^{27}$ In the present case, at variance, the $n=1$ state is the longer lived for the thickest layers. Examination of the wave functions associated with the quasistationary states helps to clarify the nature of the states and to explain the lifetime behavior.

\section{B. Wave functions}

Figure 6 presents the electronic densities of the quasistationary states $\left|\Psi\left(E_{n}, \mathbf{r}\right)\right|^{2}$ for $n=1-3$. The results are presented as a function of $d=\sqrt{2} x$ and the $z$ coordinates for $\mathbf{r}=(x, x, z)$, i.e., in a plane perpendicular to the surface and defined by the unitary vectors $\hat{e}_{d}=\left(\hat{e}_{x}+\hat{e}_{y}\right) / \sqrt{2}$ and $\hat{e}_{z}$. This plane contains the coordinate origin as well as the diagonal of the 2D unit cell, and therefore both $\mathrm{Na}^{+}$and $\mathrm{Cl}^{-}$ions in each layer. Note that for the sake of clarity, the picture span along the 


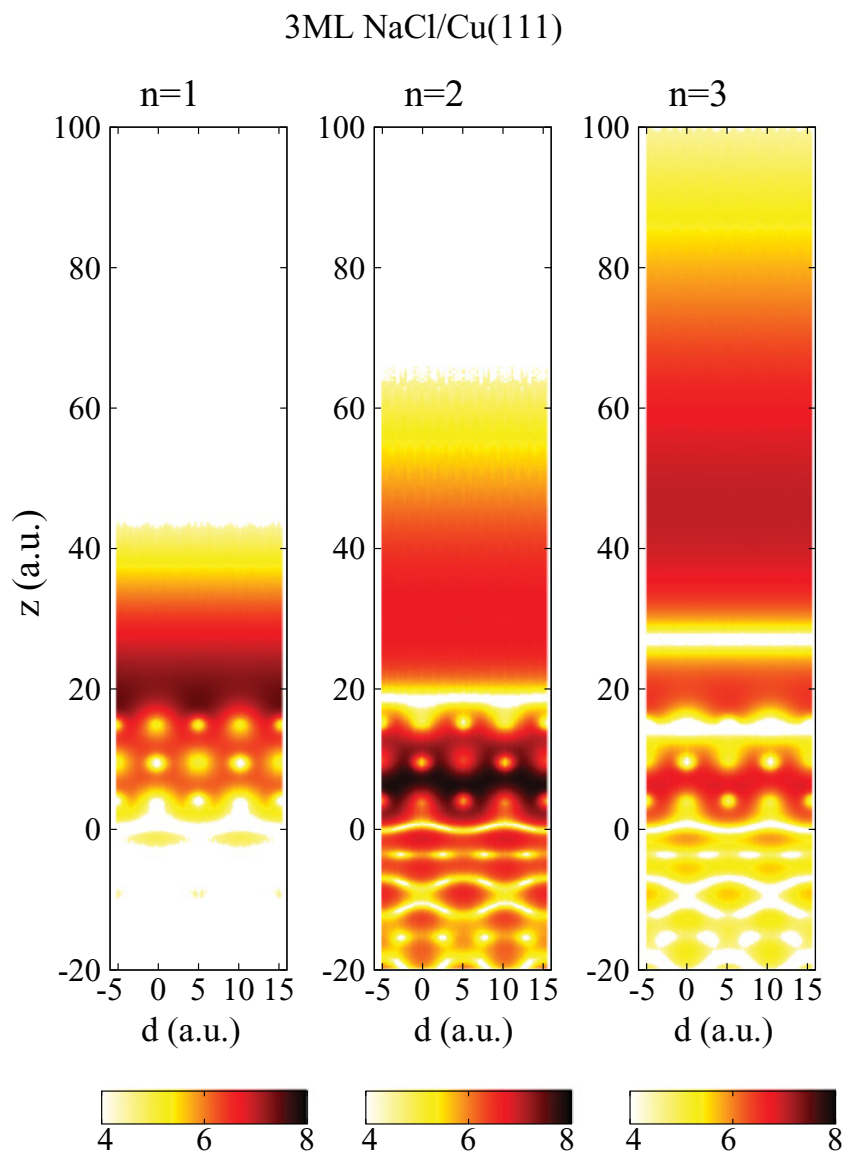

FIG. 6. (Color online) Electron density of the quasistationary states in the $3 \mathrm{ML}-\mathrm{NaCl} / \mathrm{Cu}(111)$ system. The figure presents the logarithm of the electron density for $n=1$ (left panel), $n=2$ (middle panel), and $n=3$ (right panel). Results are shown for $\mathbf{r}=(x, x, z)$ as a function of the $z$ coordinate perpendicular to the surface and the diagonal of the unit cell parallel to the surface, $d=\sqrt{2} x$. The dark areas correspond to a large probability of the presence of the electron, and the color code is given in the inset.

$d$ axis is larger by a factor 2 than the actual WPP computation box. The extension has been obtained via periodicity. The $\mathrm{NaCl}$ layer area $(0<z<20)$ is easily recognized by the light dots, corresponding to a small electron density, surrounding the center of each ion, due to the short-range repulsive potential of the ions. The metal substrate is on the negative $z$ side, and vacuum on the positive side. The density of the $n=2$ state is highly corrugated inside the metal. This is a consequence of the presence of the lattice-assisted one-electron transfer process. It is highly visible because of the interference between the $k_{\|}=0$ exponentially decreasing penetration into bulk $\mathrm{Cu}$ and the outgoing flux due to the decay into the $\mathbf{k}_{\|}=\mathbf{G}_{01}$ and $\mathbf{k}_{\|}=\mathbf{G}_{10}$ channels.

The shape of the electron density appears to be quite different for the three states. The $n=1$ and 3 states are mainly located in vacuum with a significant part inside the layer, whereas the $n=2$ state is mainly inside the layer with a significant tail in vacuum. One also recognizes the global nodes of the wave functions: no node for $n=1$, one node for $n=2$, and two nodes for $n=3$, consistent with their energy ordering. The spatial localization of the states and its evolution

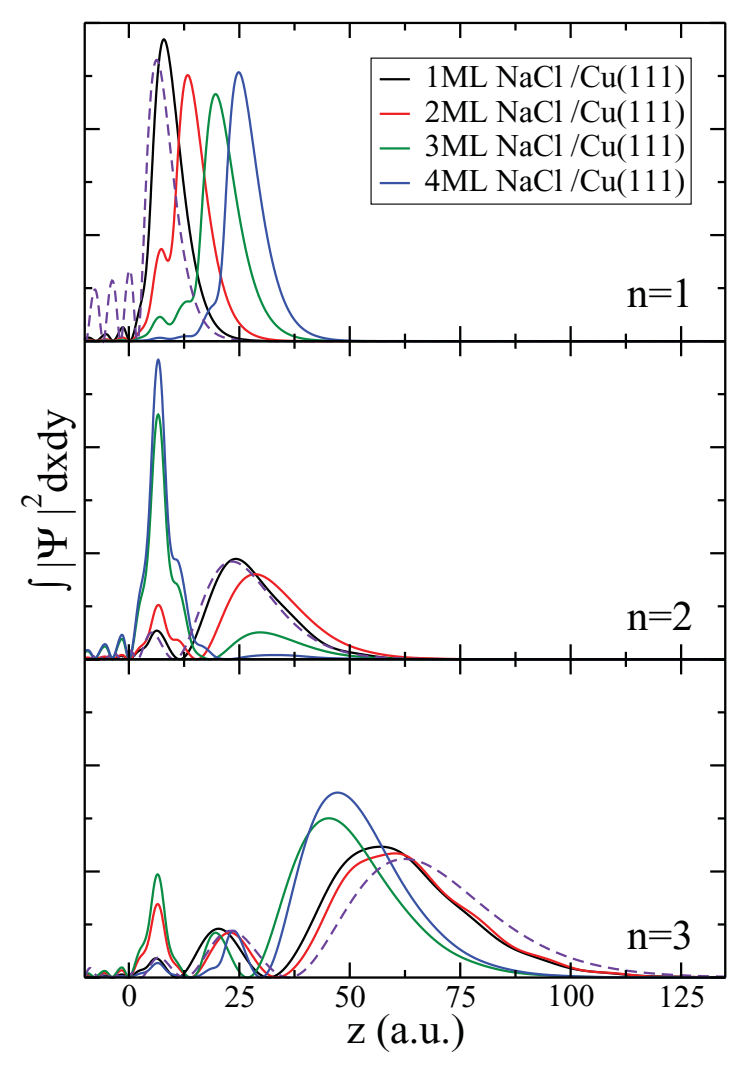

FIG. 7. (Color online) Wave functions of the $n=1,2$, and 3 resonances in the top, middle, and bottom panels, respectively, in $n \mathrm{ML}-\mathrm{NaCl} / \mathrm{Cu}(111)$. Black line, $1 \mathrm{ML}$; red line, 2ML; green line, 3ML; blue line, 4ML. The electron density $|\Psi|^{2}$ has been integrated in the surface plane $(x y)$, normalized and plotted as a function of the coordinate perpendicular to the surface, $z$. Image (resonant) states for the clean surface are shown with a dashed line.

with the layer thickness is further illustrated in Fig. 7, which presents the electron density integrated in the $(x, y)$ plane and displayed as a function of the $z$ coordinate for different $\mathrm{NaCl}$ layer thicknesses. Again, the three states appear very different, as well as their evolution with the $\mathrm{NaCl}$ layer thickness.

In Fig. 7, the $n=1$ state displays the typical behavior that is expected for the first image potential state repelled into vacuum by an insulating layer. The density in $z$ exhibits a single peak, which moves out gradually into vacuum, as the layer thickness is increased. The image potential state slightly penetrates into the layer in an exponentially decreasing way, and some corrugation due to the ionic lattice does survive the $(x, y)$ integration. As a consequence of this decrease through the layer, the penetration into bulk $\mathrm{Cu}$ is very weak and decreases with the layer thickness. This is perfectly consistent with the binding energy of the $n=1$ state with respect to vacuum that decreases when the layer increases (see Fig. 4) and with the state lifetime that increases quasiexponentially with the thickness (see Fig. 5). This view is further supported by Fig. 8, which presents $\langle z\rangle$, the mean value of $z$ over the quasistationary state wave function, as a function of the layer thickness. The mean value $\langle z\rangle$ for the $n=1$ state is seen to increase linearly with the layer thickness, actually following the position of the outer limit of the layer, in a continuous way from the clean $\mathrm{Cu}(111)$ surface case. 


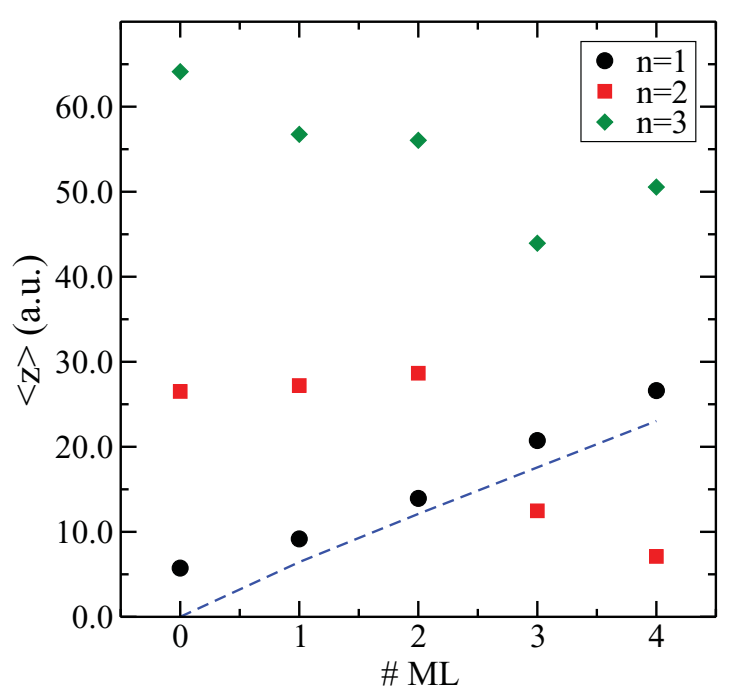

FIG. 8. (Color online) Mean value of $z$ over the quasistationary state wave function $\langle z\rangle$ in a.u., as a function of the layer thickness. Black circles, $n=1$; red squares, $n=2$; green diamonds, $n=3$. The dashed blue line represents the limit of the $\mathrm{NaCl}$ layers.

The behavior of the $n=2$ state is quite different. We can roughly separate two different situations. For 1 and 2 ML, the quasistationary state is mainly outside the layer with a little penetration inside the layer and a very weak penetration into bulk $\mathrm{Cu}$. For 3 and $4 \mathrm{ML}$, the $n=2$ state is mainly inside the layer, with a tail in vacuum and a significant penetration inside the metal. This behavior is interpreted as an effect of the conduction band of $\mathrm{NaCl}$. The bottom of the conduction band of bulk $\mathrm{NaCl}$ is at $-0.6 \mathrm{eV}$ below vacuum. ${ }^{16,17}$ This value is modified by the surface and mainly by the finite thickness of the layer. Actually, the layer behaves as a quantum well with quantization of the electron motion perpendicular to the surface inside the layer. This effect significantly raises the effective conduction band bottom for very thin layers. The $n=2$ state appears as a mixing of the second IS with the lowest state in the quantum well (the quantized state localized inside the layer with a node at each layer limit). For 1 and $2 \mathrm{ML}$, the quantum well state is very high and the $n=2$ state much resembles an IS. For 3 and 4 ML, the ground state of the quantum well is low enough and the $n=2$ state has a large component on it. This interpretation is further confirmed in Fig. 8: At 1 and 2 ML, $\langle z\rangle$ for $n=2$ is reasonable for the second IS on the surface, whereas for 3 and $4 \mathrm{ML}$, $\langle z\rangle$ is inside the layer, confirming the dominant character of the $n=2$ state as a layer state. This change of character of the $n=2$ state accounts for the drop of the level lifetime above 2ML (Fig. 5): When the state has a strong IS character it is repelled into vacuum and its lifetime is rather long; in contrast, when it has a strong conduction band state character, the electron density close to and inside bulk $\mathrm{Cu}$ is larger and the state lifetime is short.

The $n=3$ state exhibits a similar but much less marked behavior; its mixing with quantum well states is weaker, as one can see on the integrated density (Fig. 7). In addition, $\langle z\rangle$ exhibits a drop between 2 and 3 ML (Fig. 8), indicating an increased mixing with quantum well states instead of the increase in $\langle z\rangle$ expected for an IS. In a very qualitative manner, one can see that the mean value $\langle z\rangle$ for the $n=2$ state at 1 and 2 ML together with that of the $n=3$ state at 3 and $4 \mathrm{ML}$ would exhibit a reasonable behavior for a second IS on an insulating layer. Indeed, the mixing between ISs and quantum well states is very large and one cannot strictly assign the $n=2$ and 3 states to ISs or layer states. The mixing is between the Rydberg series of ISs repelled in vacuum and the series of quantum well states. In the present case, the lowest quantum well state appears diluted in the IS series. A rough estimate of the energy position of the second quantum well state using simple quantization energy puts it above the vacuum level; i.e., it is expected to be very broad and well above the present studied states. However, the fact that one of the nodes of the $n=3$ integrated density (Fig. 7) happens to be close to the layer center for $4 \mathrm{ML}$ could be an indication of a small contribution from this second quantum well state in the $n=3$ quasistationary state at $4 \mathrm{ML}$. The present situation is similar to that encountered in the case of a $\mathrm{Cu}$ quantum corral on $\mathrm{Cu}(111)$, where a quasistationary state localized on the corral wall is diluted in the series of quantized corral states. ${ }^{56}$

\section{Effective masses}

For the $3 \mathrm{ML}-\mathrm{NaCl} / \mathrm{Cu}(111)$ system, we have also evaluated the energy of the $n=1,2$, and 3 states as a function of the

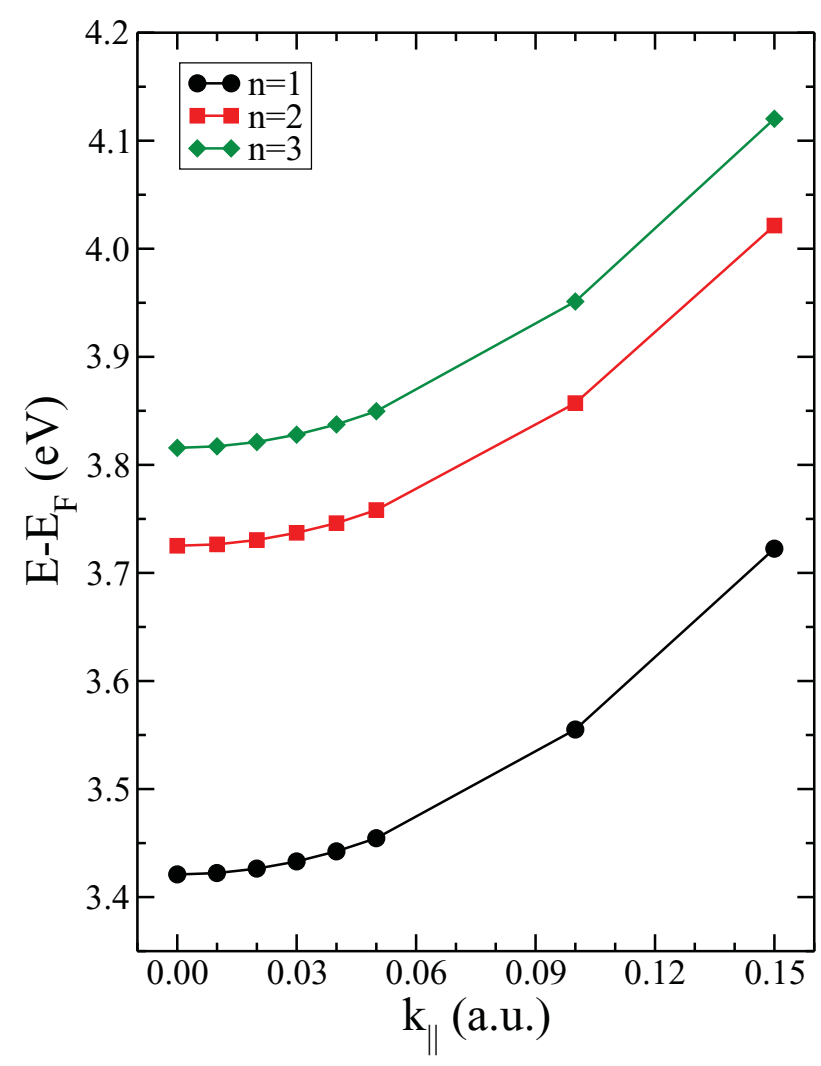

FIG. 9. (Color online) Energy (in eV) with respect to the Fermi level of the $n=1,2$, and 3 resonances in $3 \mathrm{ML}-\mathrm{NaCl} / \mathrm{Cu}(111)$ as a function of the electron momentum parallel to the surface, $k_{\|}$(in a.u.). Black circles, $n=1$; red squares, $n=2$; green diamonds, $n=3$. 
electron momentum parallel to the surface, $k_{\|}$. The results are shown in Fig. 9. They show the typical parabolic behavior:

$$
E=E_{0}+\frac{k_{\|}^{2}}{2 m^{*}},
$$

where $E_{0}$ is the band bottom (Fig. 4). The three sets of data perfectly fit the above equation and lead to values of the effective mass $m^{*}=0.985,0.962$, and 0.995 (in atomic units) for the $n=1,2$, and 3 states, respectively. This is close to the free electron mass pointing to a quasifree electron motion parallel to the surface.

\section{DISCUSSION AND CONCLUSIONS}

To summarize the above results, we found a low-lying state $(n=1)$ with the clear character of an image potential state repelled from the surface by the $\mathrm{NaCl}$ layer. The next two states have a mixed character: ISs and layer states corresponding to the quantization of the $\mathrm{NaCl}$ conduction band in the finite-size layer. The penetration of the $n=2,3$ states inside the layer leads to a severe shortening of their lifetimes, well below that of the $n=1$ state. These results are quite consistent with the Muntwiler and $\mathrm{Zhu}^{30}$ results obtained for a 2-4 ML coverage and allow more precise assignment of the observed states.

First, the $n=1$ IS does not seem to be observed in TR-2PPE. This state (except for 1ML) has a very small penetration probability into bulk $\mathrm{Cu}$, and thus a very small overlap with occupied $\mathrm{Cu}(111)$ states; it should then be difficult to photoexcite it from these states. At least, it is much more difficult to excite than the other two states, which exhibit a larger bulk penetration. In this system, there is a connection between a short lifetime and an easy photoexcitation, and the $n=2,3$ states can be expected to dominate over the $n=1$ state in a TR-2PPE experiment.

The two states observed in TR-2PPE ${ }^{30}$ are at approximately 3.98 and $3.78 \mathrm{eV}$ above $E_{F}$, i.e., in the energy range of the present $n=2,3$ states in the 2-4 ML range. The observed lifetimes measured in Ref. 30 are $110 \pm 20$ and $170 \pm 20 \mathrm{fs}$, of the order of the present lifetime for the $n=2$ state in the 3 and 4 ML cases, and shorter than that of the $n=3$ state. However, a precise correspondence between the two observed states and the present $n=2,3$ states is difficult to establish because of (i) the coexistence of several thicknesses in the experiment (the states could belong to different islands) and (ii) possible inaccuracies in the present model study (in particular, changes in the work function would result in absolute and relative energy shifts of the states and of their mixing).

Our results for the effective masses of the $n=1,2$, and 3 states slightly differ from those found in Ref. $30\left(\mathrm{~m}^{*}=\right.$ $0.8 \pm 0.1$ versus $m^{*}=0.6 \pm 0.1 m_{e}$ ).

As for the assignment of the observed states, Muntwiler and $\mathrm{Zhu}^{30}$ attribute the two states to the conduction band of the $\mathrm{NaCl}$ layers and to a surface state of the ionic crystal, of the type discussed in Refs. 57 and 58. We fully confirm the involvement of the conduction band of the $\mathrm{NaCl}$ layer in the excited states; however, we did not find any intrinsic surface state at the $\mathrm{NaCl}$ surface. In Refs. 57 and 58, these surface states are attributed to the different environment felt by the surface ions compared to bulk ions (less neighbors and a smaller Madelung potential); they appear as extensions of the bulk band and lead to states localized at the surface or to bending of the bulk bands. We did not find such states for the conduction band in the present thin layers, although the effect invoked in their formation was included. We thus attribute the states observed in TR-2PPE to a mixing between ISs and conduction band states. This assignment explains their relatively short lifetimes compared to ISs. Muntwiler and $\mathrm{Zhu}^{30}$ also observed a time dependence of the energy of the states, which they attributed to the formation of a small polaron. With the present assignment of conduction band states, we can tentatively assign the evolution of the system during the TR-2PPE experiment to a motion of the ions at the surface: An outward motion of a positive $\mathrm{Na}^{+}$ion, possibly associated with inward motions of the neighboring $\mathrm{Cl}^{-}$ions, could create an attractive patch on the surface that would bind an electron; in this process the electron in the conduction band would localize around the $\mathrm{Na}^{+}$ion due to the polarization of the medium (an ion polarization contribution). As stressed in Ref. 30, polaron formation was not observed in 3D crystals; however, the above scheme for polaron formation is a surface process and would be absent in a 3D crystal.

\section{ACKNOWLEDGMENTS}

S.D.-T. gratefully acknowledges postdoctoral support from the Triangle de la Physique and the Juan de la Cierva program from the Spanish Ministerio de Ciencia e Innovación. The calculations were partly performed with the GMPCS highperformance computer facilities of the LUMAT Federation of Orsay laboratories.

\footnotetext{
*Present address: Departamento de Química, Módulo 13, Facultad de Ciencias, Universidad Autónoma de Madrid, E-28049 Madrid, Spain; sergio.diaztendero@uam.es

†andrei.borissov@u-psud.fr

jean-pierre.gauyacq@u-psud.fr

${ }^{1}$ Photochemistry and Photophysics on Surfaces, edited by J. T. Yates and H. Petek, Chem. Rev. 106(10) (2006).

${ }^{2}$ H. Petek and S. Ogawa, Prog. Surf. Sci. 56, 239 (1997).

${ }^{3}$ X.-Y. Zhu, Surf. Sci. Rep. 56, 1 (2004).

${ }^{4}$ J. Güdde and U. Höfer, Prog. Surf. Sci. 80, 49 (2005).
}

${ }^{5}$ P. Szymanski, S. Garrett-Roe, and C. Harris, Prog. Surf. Sci. 78, 1 (2005).

${ }^{6}$ M. Wolf, E. Knoesel, and T. Hertel, Phys. Rev. B 54, R5295 (1996). ${ }^{7}$ D. F. Padowitz, W. R. Merry, R. E. Jordan, and C. B. Harris, Phys. Rev. Lett. 69, 3583 (1992).

${ }^{8}$ J. D. McNeill, J. R. L. Lingle, R. E. Jordan, D. F. Padowitz, and C. B. Harris, J. Chem. Phys. 105, 3883 (1996).

${ }^{9}$ D. C. Marinica, C. Ramseyer, A. G. Borisov, D. Teillet-Billy, J. P. Gauyacq, W. Berthold, P. Feulner, and U. Höfer, Phys. Rev. Lett. 89, 046802 (2002). 
${ }^{10}$ M. Michaud, L. Sanche, C. Gaubert, and R. Baudoing, Surf. Sci. 205, 447 (1988).

${ }^{11}$ D. C. Marinica, D. Teillet-Billy, J. P. Gauyacq, M. Michaud, and L. Sanche, Phys. Rev. B 64, 085408 (2001).

${ }^{12}$ W. Wurth and D. Menzel, Chem. Phys. 251, 141 (2000).

${ }^{13}$ J. P. Gauyacq and A. G. Borisov, Phys. Rev. B 69, 235408 (2004).

${ }^{14}$ D. C. Marinica, C. Ramseyer, A. G. Borisov, D. Teillet-Billy, and J. P. Gauyacq, Surf. Sci. 540, 457 (2003).

${ }^{15}$ M. Rohleder, W. Berthold, J. Güdde, and U. Höfer, Phys. Rev. Lett. 94, 017401 (2005).

${ }^{16}$ D. M. Roessler and W. C. Walker, Phys. Rev. 166, 599 (1968).

${ }^{17}$ G. K. Wertheim, J. E. Rowe, D. N. E. Buchanan, and P. H. Citrin, Phys. Rev. B 51, 13675 (1995).

${ }^{18}$ J. Repp and G. Meyer, Appl. Phys. A 85, 399 (2006).

${ }^{19}$ R. Bennewitz, J. Phys. Condens. Matter 18, R417 (2006).

${ }^{20}$ J. Repp, S. Fölsch, G. Meyer, and K.-H. Rieder, Phys. Rev. Lett. 86, 252 (2001).

${ }^{21}$ S. Fölsch, A. Riemann, J. Repp, G. Meyer, and K. H. Rieder, Phys. Rev. B 66, 161409 (2002).

${ }^{22}$ J. Repp, G. Meyer, S. M. Stojković, A. Gourdon, and C. Joachim, Phys. Rev. Lett. 94, 026803 (2005).

${ }^{23}$ J. Repp, G. Meyer, F. Olsson, and M. Persson, Science 305, 5683 (2004).

${ }^{24}$ J. Repp, G. Meyer, S. Paavilainen, F. Olsson, and M. Persson, Science 312, 1196 (2006).

${ }^{25}$ F. E. Olsson, S. Paavilainen, M. Persson, J. Repp, and G. Meyer, Phys. Rev. Lett. 98, 176803 (2007).

${ }^{26}$ L. Gross, F. Mohn, P. Liljeroth, J. Repp, F. Giesibl, and G. Meyer, Science 324, 1428 (2009).

${ }^{27}$ P. M. Echenique and J. B. Pendry, J. Phys. C 11, 2065 (1978).

${ }^{28}$ M. Desjonquères and D. Spanjaard, Concepts in Surface Science, Springer Verlang Series in Surface Science 40 (Springer, Berlin, 1993).

${ }^{29}$ E. V. Chulkov, A. G. Borisov, J. P. Gauyacq, D. Sánchez-Portal, V. M. Silkin, V. P. Zhukov, and P. M. Echenique, Chem. Rev. 106, 4160 (2006).

${ }^{30}$ M. Muntwiler and X.-Y. Zhu, Phys. Rev. Lett. 98, 246801 (2007).

${ }^{31}$ R. Bennewitz, V. Barwich, M. Bammerlin, C. Loppacher, M. Guggisberg, A. Baratoff, E. Meyer, and H.-J. Güntherodt, Surf. Sci. 438, 289 (1999).

${ }^{32}$ M. Pivetta, F. Patthey, M. Stengel, A. Baldereschi, and W.-D. Schneider, Phys. Rev. B 72, 115404 (2005).

${ }^{33}$ F. Olsson and M. Persson, Surf. Sci. 540, 172 (2003).

${ }^{34}$ F. E. Olsson, M. Persson, J. Repp, and G. Meyer, Phys. Rev. B 71, 075419 (2005).
${ }^{35}$ E. V. Chulkov, V. M. Silkin, and P. M. Echenique, Surf. Sci. 437, 330 (1999).

${ }^{36}$ A. G. Borisov, A. K. Kazansky, and J. P. Gauyacq, Phys. Rev. B 59, 10935 (1999).

${ }^{37}$ M. D. Feit and J. J. A. Fleck, J. Chem. Phys. 78, 301 (1983).

${ }^{38}$ R. Kosloff, J. Phys. Chem. 92, 2087 (1988).

${ }^{39}$ With the size of the cell used in our calculations, possible effects due to a lattice superstructure, namely moiré effects, have been neglected.

${ }^{40}$ A. G. Borisov et al., Phys. Rev. Lett. 101, 266801 (2008).

${ }^{41}$ M. Van Hove and W. C. C. Weinberg, Low Energy Electron Diffraction, Theory and Experiment (Springer-Verlag, Heidelberg, 1986).

${ }^{42}$ E. V. Chulkov, I. Sarría, V. M. Silkin, J. M. Pitarke, and P. M. Echenique, Phys. Rev. Lett. 80, 4947 (1998).

${ }^{43}$ T. Klamroth, P. Saalfrank, and U. Höfer, Phys. Rev. B 64, 035420 (2001).

${ }^{44}$ E. Knoesel, A. Hotzel, and M. Wolf, J. Electron Spectrosc. Related Phenom. 88-91, 577 (1998), Proceedings of the Seventh International Conference on Electron Spectroscopy.

${ }^{45}$ D. Neuhauser and M. Baer, J. Chem. Phys. 91, 4651 (1989).

${ }^{46}$ F. E. Olsson, A. G. Borisov, M. Persson, N. Lorente, A. K. Kazansky, and J. P. Gauyacq, Phys. Rev. B 70, 205417 (2004).

${ }^{47}$ S. Díaz-Tendero, S. Fölsch, F. E. Olsson, A. G. Borisov, and J. P. Gauyacq, Nano Lett. 8, 2712 (2008).

${ }^{48}$ J. N. Bardsley, Case Stud. At. Mol. Phys. 4, 299 (1974).

${ }^{49}$ G. D. Mahan, Phys. Rev. B 34, 4235 (1986).

${ }^{50}$ G. D. Mahan, Phys. Rev. B 38, 7841 (1988).

${ }^{51}$ P. Nordlander, Phys. Rev. B 46, 2584 (1992).

${ }^{52}$ R. Bennewitz, M. Bammerlin, M. Guggisberg, C. Loppacher, A. Baratoff, E. Meyer, and H. J. Güntherodt, Surf. Interface Anal. 27, 462 (1999).

${ }^{53}$ S. Prada, U. Martinez, and G. Pacchioni, Phys. Rev. B 78, 235423 (2008).

${ }^{54}$ C. Corriol, V. M. Silkin, D. Sánchez-Portal, A. Arnau, E. V. Chulkov, P. M. Echenique, T. von Hofe, J. Kliewer, J. Kröger, and R. Berndt, Phys. Rev. Lett. 95, 176802 (2005).

${ }^{55}$ S. Díaz-Tendero, F. E. Olsson, A. G. Borisov, and J.-P. Gauyacq, Phys. Rev. B 79, 115438 (2009).

${ }^{56}$ S. Díaz-Tendero, F. E. Olsson, A. G. Borisov, and J.-P. Gauyacq, Phys. Rev. B 77, 205403 (2008).

${ }^{57}$ J. D. Levine and P. Mark, Phys. Rev. 144, 751 (1966).

${ }^{58}$ W. Kisiel and B. Stankiewicz, Surf. Sci. 231, 32 (1990).

${ }^{59}$ M. Weinelt, J. Phys. Condens. Matter 14, R1099 (2002). 